UDC 54.058

\title{
TEMPERATURE DEPENDENCES OF THE PECLET NUMBER IN SUBLIMATION PROCESSES OF SIMPLE SUBSTANCES
}

\author{
A.I. Kravchenko, A.I. Zhukov, O.A. Datsenko \\ National Science Center “Kharkov Institute of Physics and Technology”, \\ Kharkiv, Ukraine \\ E-mail:krwchnko@gmail.com
}

The results of calculating the Peclet number $P e=w r /(\rho D)$ (where $D$ is the diffusion coefficient; w is the evaporation rate of the substance; $\rho$ is the density of the substance; $r$ is the size factor) in sublimation processes of simple substances with high values of vapor pressure $(\approx 1 \mathrm{~mm} \mathrm{Hg}$ and above at the melting temperature): As, Gd, $\mathrm{Tm}, \mathrm{Lu}, \mathrm{Cr}, \mathrm{Yb}, \mathrm{Sm}, \mathrm{Mg}, \mathrm{Ra}, \mathrm{Ca}, \mathrm{Sr}, \mathrm{Ni}, \mathrm{Co}, \mathrm{Eu}, \mathrm{Mn}, \mathrm{Ba}$. It is shown that the nature of the temperature dependence $P e(T)$ is determined by the properties of the components of the sublimated system "base-impurity" (including the diffusion activation energy $Q$ of impurity). For each substance for given $Q$ and $r$, the dependence $P e(T)$ is monotonic. A decrease in the process temperature can improve the purification of a substance from one of several impurities, accompanied by deterioration in the purification from another impurity.

\section{INTRODUCTION}

Sublimation is one of the methods for obtaining a number of high-purity substances, in connection with which there is an interest to the theory and practice of the method [1-9]. The advantage of the method over crystallization and distillation refining is the lower temperature of its implementation and, as a result, less contamination of the product by the container material. In this case, a special role in this method is played by the diffusion of impurities in the refined material.

A method was developed for calculating the sublimation refining of a substance, taking into account the diffusion of impurities, in which the efficiency of the process with yield $g$ is determined by two parameters (that depend on the temperature $T$ ): the separation coefficient $\beta(T)$ and the diffusion Peclet number

$$
P e(T)=\frac{w(T) r}{\rho D(T)},
$$

where $D$ is the diffusion coefficient; $w$ is the rate of evaporation of the substance from a unit surface; $\rho$ is the density of the substance; $r$ is the dimensional factor of the material (for example, the initial radius of the evaporating sphere) - Figure [10]. The cleaning efficiency of the substance increases with a decrease in the $P e$ number. (For the same substance and at the same sublimation temperature, $P e \sim r$ ). At $P e=0$, the $C / C_{0}$ dependence has the form of a distillation equation with ideal mixing of the evaporated liquid [10]:

$$
\frac{C}{C_{0}}=\frac{1-(1-g)^{\beta}}{g} \text {. }
$$

Also, a technique was developed for calculating the temperature dependence of $P e(T)$ and examples of calculating $P e(T)$ for some simple substances $(\mathrm{Mg}, \mathrm{Cr}$, $\mathrm{Sm}, \mathrm{Eu})$ in small temperature ranges near the melting point $T_{\mathrm{m}}$ are given [11]. $\beta=0.1$
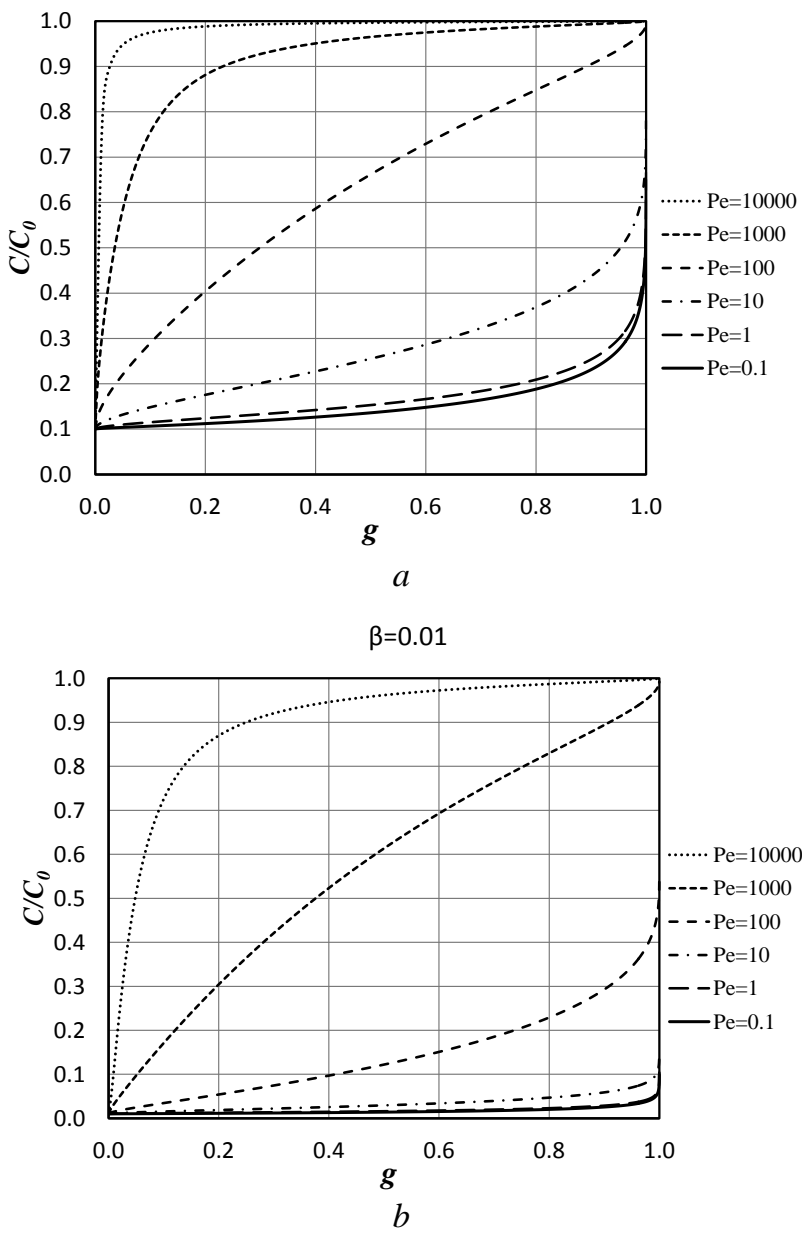

Dependence of $C / C_{0}$ on the product (condensate) from yield $g$ at different values of the Pe number and $\beta$ : $a-\beta=0.1 ; b-\beta=0.01[10]$.

$\left(C / C_{0}\right.$ is the ratio of the average impurity concentration in the condensate to the initial impurity concentration) 
Numerical determination of $P e$ values at temperature $T$ for a material in the form of a ball with an initial radius $r, \mathrm{~cm}$ was carried out according to the formula:

$$
\operatorname{Pe}(T)=\frac{0.058 p\left(\frac{M}{T}\right)^{1 / 2} r}{\rho D^{*} \exp \left[\frac{Q}{R}\left(\frac{1}{T_{m}}-\frac{1}{T}\right)\right]},
$$

where $T$ is process temperature, $\mathrm{K} ; T_{m}$ is the melting point of the substance, $\mathrm{K} ; p$ is the vapor pressure of the substance, $\mathrm{mm} \mathrm{Hg}$, at temperature $T ; M$ is the atomic mass of the substance, at. mass unit; $\rho$ is the density of the substance, $\mathrm{g} / \mathrm{cm}^{3} ; Q / R$ is the ratio of the activation energy of impurity diffusion to the universal gas constant, $\mathrm{K}$ (for most impurities in simple substances, the $Q / R$ values are in the range from $0.5 \cdot 10^{4}$ to $3 \cdot 10^{4} \mathrm{~K}$ [11]); $D^{*}$ is the diffusion coefficient, $\mathrm{cm}^{2} / \mathrm{s}$, of the impurity in the sublimated substance at temperature $T_{m}$. (For most impurities in simple substances at a temperature near $T_{m}$, the diffusion coefficient of an impurity in a liquid is $D_{m} \sim 10^{-5} \mathrm{~cm}^{2} / \mathrm{s}$, and in a solid $\left.D^{*} \sim 10^{-6} \mathrm{~cm}^{2} / \mathrm{s}[11]\right)$.

Meanwhile, it is of interest to examine in more detail the temperature dependences of $P e(T)$ : for a larger number of substances (allowing sublimation refining for practical purposes) and in an extended temperature range - to reveal patterns. This was the purpose of the work.

\section{PERFORMANCE OF CALCULATIONS}

The Peclet number in the processes of sublimation refining of simple substances with a vapor pressure of $\approx 1 \mathrm{~mm} \mathrm{Hg}$ and above at the melting temperature $T_{\mathrm{m}}$ (i.e., substances were considered, the sublimation of which can be carried out at a sufficiently high rate) was calculated. The temperatures at which the vapor pressure of the substance is greater than $0.001 \mathrm{~mm} \mathrm{Hg}$ (when a practical interest in the process remains) were considered. The calculation of the $P e$ number in a solid at a temperature near $T_{\mathrm{m}}$ (at which the diffusion coefficient is $D^{*}$ and the order of its value is known) was carried out using formula (1). To calculate $P e$ at lower temperatures, formula (2) was used. The data on the vapor pressure of substances at temperature $T$ were found from the monograph by Nesmeyanov [12]. For comparability of the results, the calculations were carried out at $r=1 \mathrm{~cm}$, and the results were denoted as $P e_{1}$. As in [11], the calculations were carried out at a value of $D^{*}=5 \cdot 10^{-6} \mathrm{~cm}^{2} / \mathrm{s}$ and three values of the activation energy of impurity diffusion. A computer program was used to perform the calculations.

\section{RESULTS AND DISCUSSION}

The calculation results are presented in Tables 1-3 (for arsenic, it is taken into account that its vapor consists mainly of $\mathrm{As}_{4}$ molecules; for comparison,
Table 1 shows the calculated $P e_{1}$ values for some substances with low vapor pressure and for two complex substances: for ice [13] and naphthalene [14]). The data in Tables 1 and 2 can be compared with the graphs in Figure.

At a temperature near $T_{m}$, for substances with a $p \approx 1 \mathrm{~mm} \mathrm{Hg}$ and higher, $P e_{1} \sim 10^{2} \ldots 10^{8}$ and for substances with a low $p, P e_{1} \approx 0$ (see Table 1).

As can be seen from the Table 2, the nature of the temperature dependence $P e_{1}(T)$ is determined by the nature of the components of the sublimated system "base-impurity". In each considered system "baseimpurity" at a given value of $Q / R$, the dependence $P e_{1}(T)$ is monotonic.

Table 1

$P e_{1}$ for substances in the solid phase at a temperature near $T_{m}\left(D=D^{*} \sim 10^{-6} \mathrm{~cm}^{2} / \mathrm{s}\right)$

\begin{tabular}{|c|c|c|c|}
\hline Substance & $T_{m}, \mathrm{~K}$ & $p, \mathrm{~mm} \mathrm{Hg}$ & $P e_{1}$ \\
\hline $\mathrm{As}$ & 1090 & $\sim 10^{5}$ & $\sim 10^{8}$ \\
$\mathrm{Gd}$ & 1586 & 189 & $\sim 10^{5}$ \\
$\mathrm{Tm}$ & 1818 & 180 & $\sim 10^{5}$ \\
$\mathrm{Lu}$ & 1936 & 19.1 & $\sim 10^{3}$ \\
$\mathrm{Cr}$ & 2130 & 7.7 & $\sim 10^{3}$ \\
$\mathrm{Sm}$ & 1350 & 4.4 & $\sim 10^{3}$ \\
$\mathrm{Yb}$ & 1097 & 3.1 & $\sim 10^{3}$ \\
$\mathrm{Mg}$ & 923 & 2.8 & $\sim 10^{4}$ \\
$\mathrm{Ra}$ & 1233 & 2.5 & $\sim 10^{4}$ \\
$\mathrm{Ca}$ & 1112 & 2.0 & $\sim 10^{4}$ \\
$\mathrm{Sr}$ & 1042 & 1.9 & $\sim 10^{4}$ \\
$\mathrm{Ni}$ & 1726 & 1.8 & $\sim 10^{2}$ \\
$\mathrm{Co}$ & 1768 & 1.4 & $\sim 10^{2}$ \\
$\mathrm{Eu}$ & 1099 & 1.1 & $\sim 10^{4}$ \\
$\mathrm{Mn}$ & 1517 & 0.9 & $\sim 10^{2}$ \\
$\mathrm{Ba}$ & 1002 & 0.8 & $\sim 10^{3}$ \\
\hline $\mathrm{Fe}$ & 1812 & $\sim 10^{-2}$ & $\sim 1$ \\
$\mathrm{Cu}$ & 1357 & $\sim 10^{-4}$ & $\sim 10^{-2}$ \\
$\mathrm{Al}$ & 934 & $\sim 10^{-9}$ & $\sim 10^{-6}$ \\
$\mathrm{In}$ & 430 & $\sim 10^{-19}$ & $\sim 10^{-17}$ \\
$\mathrm{Ga}$ & 303 & $\sim 10^{-38}$ & $\sim 10^{-34}$ \\
\hline $\mathrm{C}_{10} \mathrm{H}_{8}$ & 353 & 8 & $\sim 10^{4}$ \\
$\mathrm{H}_{2} \mathrm{O}$ & 273 & 5 & $\sim 10^{4}$ \\
\hline
\end{tabular}


Table 2

End of Table 2

Dependence $P e_{1}(T)$ for various substances and

impurities with different values of the diffusion activation energy $\left(T_{m}, \mathrm{~K}\right.$, of the substance is indicated near the chemical symbols)

\begin{tabular}{|c|c|c|c|c|c|}
\hline \multirow{2}{*}{$\begin{array}{l}\text { Sub- } \\
\text { stance }\end{array}$} & \multirow{2}{*}{$\begin{array}{l}T, \\
\mathrm{~K}\end{array}$} & \multirow{2}{*}{$\begin{array}{c}p \\
\mathrm{~mm} \mathrm{Hg}\end{array}$} & \multicolumn{3}{|c|}{$\begin{array}{c}P e_{1} \\
\text { at different values of } Q / R\end{array}$} \\
\hline & & & $1 \cdot 10^{4} \mathrm{~K}$ & $2 \cdot 10^{4} \mathrm{~K}$ & $3 \cdot 10^{4} \mathrm{~K}$ \\
\hline \multirow{5}{*}{$\begin{array}{c}\text { As } \\
1090\end{array}$} & 600 & 0.15 & $\sim 10^{5}$ & \begin{tabular}{|c|} 
\\
$\sim 10^{8}$
\end{tabular} & $\sim 10^{12}$ \\
\hline & 700 & 7.3 & $\sim 10^{6}$ & $\sim 10^{8}$ & $\sim 10^{10}$ \\
\hline & 800 & 121 & $\sim 10^{6}$ & $\sim 10^{8}$ & $\sim 10^{9}$ \\
\hline & 900 & $\sim 10^{3}$ & $\sim 10^{6}$ & $\sim 10^{7}$ & $\sim 10^{8}$ \\
\hline & 1000 & $\sim 10^{4}$ & $\sim 10^{7}$ & $\sim 10^{7}$ & $\sim 10^{8}$ \\
\hline \multirow{5}{*}{$\begin{array}{c}\mathrm{Gd} \\
1586\end{array}$} & 1000 & 0.01 & 328 & $1 \cdot 10^{4}$ & $5 \cdot 10^{5}$ \\
\hline & 1200 & 1 & $4 \cdot 10^{3}$ & $3 \cdot 10^{4}$ & $2 \cdot 10^{5}$ \\
\hline & 1300 & 5 & $1 \cdot 10^{4}$ & $4 \cdot 10^{4}$ & $2 \cdot 10^{5}$ \\
\hline & 1400 & 20 & $2 \cdot 10^{4}$ & $5 \cdot 10^{4}$ & $1 \cdot 10^{5}$ \\
\hline & 1500 & 67 & $5 \cdot 10^{4}$ & $7 \cdot 10^{4}$ & $9 \cdot 10^{4}$ \\
\hline \multirow{8}{*}{$\begin{array}{c}\mathrm{Tm} \\
1818\end{array}$} & 1100 & $\sim 10^{-3}$ & 102 & $4 \cdot 10^{3}$ & $1.4 \cdot 10^{5}$ \\
\hline & 1200 & 0.05 & 414 & $7 \cdot 10^{3}$ & $1.2 \cdot 10^{5}$ \\
\hline & 1300 & 0.3 & $1 \cdot 10^{3}$ & $1 \cdot 10^{4}$ & $1.1 \cdot 10^{5}$ \\
\hline & 1400 & 2 & $4 \cdot 10^{3}$ & $2 \cdot 10^{4}$ & $1.0 \cdot 10^{5}$ \\
\hline & 1500 & 6 & $9 \cdot 10^{3}$ & $3 \cdot 10^{4}$ & $9 \cdot 10^{4}$ \\
\hline & 1600 & 21 & $2 \cdot 10^{4}$ & $4 \cdot 10^{4}$ & $8 \cdot 10^{4}$ \\
\hline & 1700 & 62 & $4 \cdot 10^{4}$ & $5 \cdot 10^{4}$ & $8 \cdot 10^{4}$ \\
\hline & 1800 & 180 & $7 \cdot 10^{4}$ & $8 \cdot 10^{4}$ & $8 \cdot 10^{4}$ \\
\hline \multirow{6}{*}{$\begin{array}{c}\mathrm{Lu} \\
1936\end{array}$} & 1400 & $\sim 10^{-3}$ & 18 & 131 & 947 \\
\hline & 1500 & 0.04 & 73 & 326 & $1.5 \cdot 10^{3}$ \\
\hline & 1600 & 0.2 & 232 & 685 & $2.0 \cdot 10^{3}$ \\
\hline & 1700 & 0.7 & 545 & $1 \cdot 10^{3}$ & $2.3 \cdot 10^{3}$ \\
\hline & 1800 & 2 & $1 \cdot 10^{3}$ & $2 \cdot 10^{3}$ & $2.9 \cdot 10^{3}$ \\
\hline & 1900 & 7 & $3 \cdot 10^{3}$ & $3 \cdot 10^{3}$ & $4 \cdot 10^{3}$ \\
\hline \multirow{5}{*}{$\begin{array}{c}\mathrm{Cr} \\
2130\end{array}$} & 1600 & $\sim 10^{-3}$ & 9 & 33 & 154 \\
\hline & 1700 & 0.03 & 28 & 91 & 298 \\
\hline & 1800 & 0.1 & 65 & 153 & 362 \\
\hline & 1900 & 0.4 & 188 & 332 & 587 \\
\hline & 2000 & 1 & 458 & 622 & 844 \\
\hline \multirow{4}{*}{$\begin{array}{c}\mathrm{Sm} \\
1350\end{array}$} & 1000 & $\sim 10^{-3}$ & 78 & $1 \cdot 10^{3}$ & $1 \cdot 10^{4}$ \\
\hline & 1100 & 0.09 & 289 & $2 \cdot 10^{3}$ & $8 \cdot 10^{3}$ \\
\hline & 1200 & 0.9 & $1 \cdot 10^{3}$ & $3 \cdot 10^{3}$ & $8 \cdot 10^{3}$ \\
\hline & 1300 & 3 & $2 \cdot 10^{3}$ & $3 \cdot 10^{3}$ & $4 \cdot 10^{3}$ \\
\hline \multirow{3}{*}{$\begin{array}{c}\mathrm{Yb} \\
1097\end{array}$} & 800 & $\sim 10^{-3}$ & 91 & $2.7 \cdot 10^{3}$ & $8 \cdot 10^{4}$ \\
\hline & 900 & 0.06 & 321 & $2.4 \cdot 10^{3}$ & $2 \cdot 10^{4}$ \\
\hline & 1000 & 0.5 & 834 & $2.0 \cdot 10^{3}$ & $5 \cdot 10^{3}$ \\
\hline \multirow{3}{*}{$\begin{array}{l}\mathrm{Mg} \\
923\end{array}$} & 700 & $\sim 10^{-3}$ & 281 & $9 \cdot 10^{3}$ & $8 \cdot 10^{5}$ \\
\hline & 800 & 0.2 & $1 \cdot 10^{3}$ & $7 \cdot 10^{3}$ & $4 \cdot 10^{4}$ \\
\hline & 900 & 1.7 & $3 \cdot 10^{3}$ & $3 \cdot 10^{3}$ & $4 \cdot 10^{3}$ \\
\hline \multirow{5}{*}{$\begin{array}{c}\mathrm{Ra} \\
1233\end{array}$} & 800 & $\sim 10^{-3}$ & 442 & $4 \cdot 10^{4}$ & $9 \cdot 10^{6}$ \\
\hline & 900 & 0.05 & $1 \cdot 10^{3}$ & $3 \cdot 10^{4}$ & $5 \cdot 10^{5}$ \\
\hline & 1000 & 0.4 & $3 \cdot 10^{3}$ & $2 \cdot 10^{4}$ & $1 \cdot 10^{5}$ \\
\hline & 1100 & 2 & $6 \cdot 10^{3}$ & $2 \cdot 10^{4}$ & $4 \cdot 10^{4}$ \\
\hline & 1200 & 7 & $1 \cdot 10^{4}$ & $1 \cdot 10^{4}$ & $2 \cdot 10^{4}$ \\
\hline \multirow{4}{*}{$\begin{array}{c}\mathrm{Ca} \\
1112\end{array}$} & 800 & $\sim 10^{-3}$ & 56 & $2 \cdot 10^{3}$ & $6 \cdot 10^{4}$ \\
\hline & 900 & 0.01 & 131 & $1 \cdot 10^{3}$ & $9 \cdot 10^{3}$ \\
\hline & 1000 & 0.1 & 410 & $1 \cdot 10^{3}$ & $3 \cdot 10^{3}$ \\
\hline & 1100 & 6 & $9 \cdot 10^{3}$ & $1 \cdot 10^{4}$ & $1 \cdot 10^{4}$ \\
\hline \multirow{3}{*}{$\begin{array}{c}\mathrm{Sr} \\
1042\end{array}$} & 800 & $\sim 10^{-3}$ & 224 & $4 \cdot 10^{3}$ & $7 \cdot 10^{4}$ \\
\hline & 900 & 0.1 & 658 & $3 \cdot 10^{3}$ & $1 \cdot 10^{4}$ \\
\hline & 1000 & 0.9 & $2 \cdot 10^{3}$ & $3 \cdot 10^{3}$ & $4 \cdot 10^{3}$ \\
\hline
\end{tabular}

\begin{tabular}{|c|c|c|c|c|c}
\hline \multirow{2}{*}{$\begin{array}{c}\text { Sub- } \\
\text { stance }\end{array}$} & \multirow{2}{*}{$T}$, & \multirow{2}{*}{$\begin{array}{c}\text { } \\
\mathrm{K}\end{array}$} & $\mathrm{m}$ mg & \multicolumn{3}{|c}{$\mathrm{Pe}_{1}$} \\
\cline { 4 - 6 } & & & $1 \cdot 10^{4} \mathrm{~K}$ & $2 \cdot 10^{4} \mathrm{~K}$ & $3 \cdot 10^{4} \mathrm{~K}$ \\
\hline $\mathrm{Ni}$ & 1300 & $\sim 10^{-3}$ & 4 & 25 & 165 \\
1726 & 1400 & $9 \cdot 10^{-3}$ & 9 & 36 & 138 \\
& 1500 & 0.06 & 37 & 89 & 213 \\
& 1600 & 0.3 & 118 & 187 & 295 \\
& 1700 & 1.3 & 345 & 377 & 412 \\
\hline $\mathrm{Co}$ & 1300 & $\sim 10^{-3}$ & 4 & 33 & 250 \\
1768 & 1400 & $9 \cdot 10^{-3}$ & 11 & 47 & 208 \\
& 1500 & 0.02 & 43 & 117 & 322 \\
& 1600 & 0.1 & 136 & 246 & 446 \\
& 1700 & 0.7 & 396 & 496 & 622 \\
\hline $\mathrm{Eu}$ & 900 & 0.02 & 137 & $1 \cdot 10^{3}$ & $8 \cdot 10^{3}$ \\
1099 & 1000 & 0.2 & 428 & $1 \cdot 10^{3}$ & $3 \cdot 10^{3}$ \\
\hline $\mathrm{Mn}$ & 1200 & $\sim 10^{-3}$ & 8 & 44 & 254 \\
1517 & 1300 & 0.03 & 30 & 89 & 267 \\
& 1400 & 0.2 & 110 & 190 & 330 \\
& 1500 & 0.8 & 264 & 284 & 306 \\
\hline $\mathrm{Ba}$ & 900 & 0.01 & 40 & 124 & 385 \\
1002 & 1000 & 0.1 & 125 & 128 & 130 \\
\hline & & & & \multicolumn{2}{|c}{ Table 3 }
\end{tabular}

Comparison of $P e_{1}\left(0.5 T_{\mathrm{m}}\right)$ and $P e_{1}\left(T_{\mathrm{m}}\right)$ at different values of the activation energy of impurity diffusion

\begin{tabular}{|c|c|c|c|c|c|}
\hline \multirow{2}{*}{$\begin{array}{c}\text { Sub- } \\
\text { stance }\end{array}$} & $\approx T_{m}$ & $\approx 0.5 T_{m}$ & \multicolumn{3}{|c|}{$P e_{1}\left(0.5 T_{\mathrm{m}}\right) / P e_{1}\left(T_{\mathrm{m}}\right)$} \\
\cline { 4 - 6 } & & & $1 \cdot 10^{4} \mathrm{~K}$ & $2 \cdot 10^{4} \mathrm{~K}$ & $3 \cdot 10^{4} \mathrm{~K}$ \\
\hline $\mathrm{As}$ & 1000 & 600 & $\sim 10^{-2}$ & $\sim 10$ & $\sim 10^{4}$ \\
$\mathrm{Gd}$ & 1500 & 800 & $\sim 10^{-3}$ & $\sim 1$ & $\sim 10$ \\
$\mathrm{Tm}$ & 1800 & 900 & $\sim 10^{-4}$ & $\sim 10^{-3}$ & $\sim 1$ \\
$\mathrm{Lu}$ & 1900 & 1000 & $\sim 10^{-6}$ & $\sim 10^{-4}$ & $\sim 0.1$ \\
$\mathrm{Cr}$ & 2000 & 1000 & $\sim 10^{-7}$ & $\sim 10^{-5}$ & $\sim 10^{-4}$ \\
$\mathrm{Sm}$ & 1300 & 700 & $\sim 10^{-5}$ & $\sim 0.1$ & $\sim 10^{2}$ \\
$\mathrm{Yb}$ & 1000 & 700 & $\sim 10^{-2}$ & $\sim 1$ & $\sim 10^{2}$ \\
$\mathrm{Mg}$ & 900 & 500 & $\sim 10^{-3}$ & $\sim 10$ & $\sim 10^{5}$ \\
$\mathrm{Ra}$ & 1200 & 600 & $\sim 10^{-4}$ & $\sim 1$ & $\sim 10$ \\
$\mathrm{Ca}$ & 1100 & 600 & $\sim 10^{-4}$ & $\sim 0.1$ & $\sim 10^{3}$ \\
$\mathrm{Sr}$ & 1000 & 500 & $\sim 10^{-4}$ & $\sim 1$ & $\sim 10^{4}$ \\
$\mathrm{Ni}$ & 1700 & 900 & $\sim 10^{-7}$ & $\sim 10^{-5}$ & $\sim 10^{-3}$ \\
$\mathrm{Co}$ & 1700 & 900 & $\sim 10^{-7}$ & $\sim 10^{-5}$ & $\sim 10^{-3}$ \\
$\mathrm{Eu}$ & 1000 & 600 & $\sim 10^{-3}$ & $\sim 0.1$ & $\sim 10^{2}$ \\
$\mathrm{Mn}$ & 1500 & 800 & $\sim 10^{-6}$ & $\sim 10^{-4}$ & $\sim 0.1$ \\
$\mathrm{Ba}$ & 1000 & 500 & $\sim 10^{-5}$ & $\sim 0.1$ & $\sim 10^{4}$ \\
\hline
\end{tabular}

\section{CONCLUSIONS}

The Peclet number $(\mathrm{Pe})$ was considered as one of the two main parameters of sublimation refining (the purification efficiency increases with a decrease in the Peclet number). At various values of the process temperature and the activation energy of the impurity diffusion, the Peclet numbers were calculated for a number of simple substances with high vapor pressures $(\approx 1 \mathrm{~mm} \mathrm{Hg}$ and higher at the melting point of the substance): As, Gd, Tm, Lu, Cr, Yb, Sm, Mg, Ra, Ca, $\mathrm{Sr}, \mathrm{Ni}, \mathrm{Co}, \mathrm{Eu}, \mathrm{Mn}, \mathrm{Ba}$. For comparability of the results, the calculations were performed for a material in the form of a ball with a unit initial value of the radius at a given value of the diffusion coefficient of an impurity in a solid near the melting point $D^{*}=5 \cdot 10^{-6} \mathrm{~cm}^{2} / \mathrm{s}$. It was found that the nature of the $P e_{1}(T)$ dependence is 
determined by the properties of the components of the "base-impurity" system (including the activation energy of impurity diffusion): a decrease in the process temperature can improve the purification of a substance from one of several impurities, accompanied by a deterioration in the purification from another impurity.

A simple possibility of estimating the Peclet number for materials in the solid phase at a temperature near the melting point when $P e=w r /\left(\rho D^{*}\right)$ is noted - Table 1 .

In the general case, to clarify the dependence of $C / C_{0}$ from $T$ in the sublimation process with given $g$, it is necessary to take into account both $\beta(T)$ and $P e(T)$.

\section{REFERENCES}

1. E. Ignatovich. Chemical Engineering. Processes and apparatus. M.: "Tekhnosfera", 2007, 656 p.

2. Yu.I. Dytnerskii. Processes and apparatus of chemical technology: Textbook for high schools. P. 2. Mass exchange processes and apparatus. M.: "Himiya", 1995, 368 p.

3. A.I. Kravchenko. Relationship between Effective and Ideal Separation Factors for Distillation and Sublimation // Inorganic Materials. 2016, v. 52, N 4, p. 378-385.

4. A.I. Kravchenko. Separation factors at sublimation refining of some lanthanides // Problems of Atomic Science and Technology. 2020, N 1, p. 14-16.

5. L.A. Nisel'son, A.G. Yaroshevskii, A.A. Gasanov, K.V. Tret'yakova. Glubokaya ochistka mysh'yaka (Deep cleaning of arsenic) // Vysokochistye veshchestva. 1993, N 4, p. 62-74 (in Russian).

6. A.M. Ionov, T.V. Nikiforova, N.N. Rytus. Aspects of the purification of volatile rare earth metals by UHV sublimation: Sm, Eu, Tm, Yb // Vacuum. 1996, v. 47, N 6-8, p. 879-793.

7. I.I. Papirov, A.I. Kravchenko, A.V. Shiyan, A.I. Mazin. Removal of low-volatile impurities from magnesium by sublimation // Inorganic materials. 2014, v. 50, N 5, p. 452-454.

8. I.I. Papirov, A.I. Kravchenko, A.I. Mazin, A.V. Shiyan, V.D. Virich. Impurity distribution in a magnesium sublimate // Inorganic materials. 2015, v. 51, N 6, p. 563-565.

9. I.I. Papirov, A.I. Kravchenko, A.I. Mazin, A.V. Shiyan, V.D. Virich. Impurity distributions in a magnesium sublimates // Problems of Atomic Science and Technology. 2016, N 1, p. 21-22.

10. A.I. Zhukov, A.I. Kravchenko. Calculation of sublimation with taking into account of diffusion of impurity // Inorganic materials. 2017, v. 53, N 6, p. 648-653.

11. A.I. Kravchenko, A.I. Zhukov. Temperature dependence of the Peclet diffusion number in the processes of sublimation of some simple substances // Inorganic materials. 2021, v. 57, N 7, p. 753-759.

12. A.N. Nesmeyanov. Vapor Pressure of Chemical Elements. M.: “Akad. Nauk SSSR”, 1961, 396 p.

13. A.L. Buck. New equations for computing vapor pressure and enhancement factor // J. of Applied Meteorology and Climatology. 1981, v. 20, p. 15271533.

14. New handbook of chemist and technologist. Radioactive substances. Harmful substances. Hygiene standards / Ed. board: Moskvitin A.V. and others. SPb.: ANO NPO "Professional", 2004, 1142 p.

Article received 12.05.2021

\title{
ТЕМПЕРАТУРНЫЕ ЗАВИСИМОСТИ ЧИСЛА ПЕКЛЕ В СУБЛИМАЦИОННЫХ ПРОЦЕССАХ РАФИНИРОВАНИЯ ПРОСТЫХ ВЕЩЕСТВ
}

\author{
А.И. Кравченко, А.И. Жуков, О.А. Даценко
}

Представлены результаты вычислений числа Пекле $P e=w r /(\rho D)$ (где $D-$ коэффициент диффузии; $w-$ скорость испарения вещества; $\rho$ - плотность вещества; $r$ - размерный фактор) в процессах сублимации простых веществ с высокими значениями давления пара ( $\approx 1$ мм рт. ст. и выше при температуре плавления): $\mathrm{As}, \mathrm{Gd}, \mathrm{Tm}, \mathrm{Lu}, \mathrm{Cr}, \mathrm{Yb}, \mathrm{Sm}, \mathrm{Mg}, \mathrm{Ra}, \mathrm{Ca}, \mathrm{Sr}, \mathrm{Ni}, \mathrm{Co}, \mathrm{Eu}, \mathrm{Mn}$, Ва. Показано, что характер температурной зависимости $P e(T)$ определяется свойствами компонентов сублимируемой системы «основа-примесь» (включая энергию активации диффузии $Q$ примеси). Для каждого вещества при заданных $Q$ и $r$ зависимость $P e(T)$ монотонна. Снижение температуры процесса может улучшать очистку вещества от одной из нескольких примесей, сопровождаясь ухудшением очистки от другой примеси.

\section{ТЕМПЕРАТУРНІ ЗАЛЕЖНОСТІ ЧИСЛА ПЕКЛЕ В СУБЛІМАЦЙНИХ ПРОЦЕСАХ РАФІНУВАННЯ ПРОСТИХ РЕЧОВИН}

\author{
О.І. Кравченко, О.І. Жуков, О.А. Даценко
}

Представлено результати обчислень числа Пекле $P e=w r /(\rho D)$ (де $D-$ коефіцієнт дифузії; $w$ - швидкість випаровування речовини; $\rho$ - щільність речовини; $r$ - розмірний фактор) у процесах сублімації простих речовин $з$ високими значеннями тиску пари ( $\approx 1$ мм рт. ст. і вище при температурі плавлення): $\mathrm{As}, \mathrm{Gd}, \mathrm{Tm}$, $\mathrm{Lu}, \mathrm{Cr}, \mathrm{Yb}, \mathrm{Sm}, \mathrm{Mg}, \mathrm{Ra}, \mathrm{Ca}, \mathrm{Sr}, \mathrm{Ni}, \mathrm{Co}, \mathrm{Eu}, \mathrm{Mn}, \mathrm{Ba}$. Показано, що характер температурної залежності $P е(T)$ визначається властивостями компонентів системи «основа-домішка» (включаючи енергію активації дифузії $Q$ домішки). Для кожної речовини при заданих $Q$ і $r$ залежність $P e(T)$ монотонна. Зниження температури процесу може покращувати очищення речовини від однієї 3 кількох домішок, супроводжуючись погіршенням очищення від іншої домішки. 